\title{
Nonanalytic automorphic integrals on the Hecke groups
}

\author{
by \\ Paul C. Pasles (Villanova, PA)
}

1. Introduction. Since its genesis over a century ago in work of Jacobi, Riemann, Poincaré and Klein [Ja29, Ri53, Le64], the theory of automorphic forms has burgeoned from a branch of analytic number theory into an industry all its own. Natural extensions of the theory are to integrals [Ei57, Kn94a, KS96, Sh94], thereby encompassing Hurwitz's prototype, the analytic weight 2 Eisenstein series [Hu81], and to nonanalytic forms [He59, Ma64, Sel56, ER74, Fr85]. A generalization in both directions at once has also been the subject of some scrutiny. In the present study, inspired by unpublished work of Knopp [Kn94], we consider the nonanalytic automorphic integral.

2. Some definitions. We will use standard notation: $\mathbb{Z}, \mathbb{R}$, and $\mathbb{C}$ are the sets of integer, real, and complex numbers, respectively; $\mathcal{H}$ denotes the upper half-plane $\{z \in \mathbb{C}: \operatorname{Im} z>0\}$; and $\operatorname{SL}(2 ; \mathbb{R})$ is the group of real invertible $2 \times 2$ matrices. An action of $\operatorname{SL}(2 ; \mathbb{R})$ on $\mathcal{H}$ is defined by

$$
V z=\frac{\alpha z+\beta}{\gamma z+\delta}, \quad V=\left[\begin{array}{cc}
\alpha & \beta \\
\gamma & \delta
\end{array}\right] \in \mathrm{SL}(2 ; \mathbb{R})
$$

(Thus $V z=(-V) z$.) We will be concerned with a special family of subgroups of $\mathrm{SL}(2 ; \mathbb{R})$ : For $\lambda>0$, the Hecke group is $\mathcal{G}_{\lambda}=\left\langle S_{\lambda}, T\right\rangle$, where

$$
S_{\lambda}=\left[\begin{array}{cc}
1 & \lambda \\
0 & 1
\end{array}\right] \text { and } T=\left[\begin{array}{cc}
0 & -1 \\
1 & 0
\end{array}\right]
$$

Observe that $S_{\lambda}=z+\lambda, T z=-1 / z$. It is a well-known fact that $\mathcal{G}_{\lambda}$ is (topologically) discrete if and only if $\lambda \geq 2$ or $\lambda=2 \cos (\pi / n), n=3,4,5, \ldots$

1991 Mathematics Subject Classification: Primary 11F12; Secondary 11F11, 11F25, $11 \mathrm{~F} 37$.

Key words and phrases: automorphic integrals, modular integrals, weight-changing operators, Eisenstein series.

Special thanks to my thesis advisor, Professor Marvin Knopp. 
[He38, Ha99]. Special cases: $\mathcal{G}_{1}$ is called the modular group, $\mathcal{G}_{2}$ the theta group.

For $z, w \in \mathbb{C}, z \neq 0$, define exponentiation by $z^{w}=e^{w \log z}$; here $\log z=$ $\log |z|+i \arg z$, where $\log |z|$ represents the principal branch $(\log 1=0)$, and $\arg z$ is taken in the interval $[-\pi, \pi)$. We will make exceptions to this "unitary" argument convention in certain circumstances where it will be carefully noted that we observe a "binary" convention, to be explained presently.

It will be convenient for us to consider certain functions on the Hecke groups which are analogous to a group character. We call $v: \mathcal{G}_{\lambda} \rightarrow \mathbb{C}$ a multiplier system on $\mathcal{G}_{\lambda}$ of coweights $\alpha, \beta \in \mathbb{C}$ if $\left|v\left(S_{\lambda}\right)\right|=1, v(T) \neq 0$ and $v$ satisfies the consistency condition

$$
\begin{aligned}
& v\left(M_{3}\right)\left(c_{3} z+d_{3}\right)^{\alpha}\left(c_{3} \bar{z}+d_{3}\right)^{\beta} \\
& \quad=v\left(M_{1}\right)\left(c_{1} M_{2} z+d_{1}\right)^{\alpha}\left(c_{1} M_{2} \bar{z}+d_{1}\right)^{\beta} v\left(M_{2}\right)\left(c_{2} z+d_{2}\right)^{\alpha}\left(c_{2} \bar{z}+d_{2}\right)^{\beta}
\end{aligned}
$$

for all $M_{1}, M_{2} \in \mathcal{G}_{\lambda}, M_{1} M_{2}=M_{3}, M_{j}=\left[\begin{array}{ll}a_{j} & b_{j} \\ c_{j} & d_{j}\end{array}\right]$ for $j=1,2,3, z \in \mathcal{H}$, where we interpret the consistency condition according to the binary argument convention:

$$
-\pi \leq \arg (c z+d)<\pi, \quad-\pi<\arg (c \bar{z}+d) \leq \pi,
$$

for $z \in \mathcal{H}, c, d$ not both zero.

The binary convention guarantees that whenever $c, d \in \mathbb{R}$ and $z \in \mathcal{H}$, it follows that $\arg (c \bar{z}+d)=-\arg (c z+d)$ and therefore that $\log (c \bar{z}+d)+$ $\log (c z+d) \in \mathbb{R}$. The seemingly cumbersome dichotomy, which derives from the Petersson-Maass tradition (e.g. [Ma64]), will prove convenient in many instances. For example, it implies that $v$ is a multiplier system on $\mathcal{G}_{\lambda}$ of coweights $\alpha, \beta$ if and only if $v$ is a multiplier system on $\mathcal{G}_{\lambda}$ of coweights $\alpha+\omega+2 k, \beta+\omega+2 l$ for all $k, l \in \mathbb{Z}, \omega \in \mathbb{C}[\mathrm{Pa} 98]$.

For the remainder of this work we focus mainly on the case $v\left(S_{\lambda}\right)=1$.

Definition 2.1. Let $\left\{a_{n}\right\}_{n=0}^{\infty}$ be a sequence of complex numbers with (at worst) polynomial growth in $n$. Put

$$
f(z)=\sum_{n=0}^{\infty} a_{n} e^{2 \pi i n z / \lambda}
$$

for $z \in \mathcal{H}$. Let $v$ be a multiplier system on $\mathcal{G}_{\lambda}$ of real coweights $k, 0$ with $v\left(S_{\lambda}\right)=1$. If $f$ satisfies the transformation law

$$
z^{-k} f(-1 / z)=v(T) f(z)+q(z)
$$

for all $z \in \mathcal{H}$, where

$$
q(z)=\sum_{j=1}^{J} z^{\alpha_{j}} \sum_{t=0}^{T} \beta_{j, t}(\log z)^{t},
$$


$\alpha_{j}, \beta_{j, t} \in \mathbb{C}$, we say that $f$ is an automorphic integral of coweights $k, 0$ and multiplier system $v$ on $\mathcal{G}_{\lambda}$. The function $q(z)$ is called a log-polynomial sum (term coined by D. Zeilberger).

An analytic automorphic integral is thus defined as a Fourier series, which may be viewed instead as a power series in an exponential variable. Often one allows a Laurent expansion here, at least in the case $q \equiv 0$, so that there are poles at the cusps of the fundamental region (e.g. Klein's $J$ [Ap90]), and some authors define a form so as to allow poles in $\mathcal{H}$ (e.g. [Kn93]), but we will generalize in a different direction. Instead we shall relax the analyticity condition entirely.

Definition 2.2. Let $\left\{a_{n_{1}, n_{2}, m} \mid 0 \leq n_{1}, n_{2}<\infty, 1 \leq m \leq M\right\}$ be a sequence of complex numbers satisfying

$$
\sum_{n_{1}+n_{2}=n} a_{n_{1}, n_{2}, m}=\mathcal{O}\left(n^{\gamma}\right), \quad \gamma>0, \text { as } n \rightarrow \infty .
$$

Put

$$
f(z)=\sum_{m=1}^{M} \sum_{n_{1}, n_{2}=0}^{\infty} y^{w_{m}} a_{n_{1}, n_{2}, m} e^{(2 \pi i / \lambda)\left(n_{1} z-n_{2} \bar{z}\right)},
$$

$z=x+i y \in \mathcal{H}$. (Here $w_{1}, \ldots, w_{M}$ are complex numbers.) Let $v$ be a multiplier system on $\mathcal{G}_{\lambda}$ of coweights $\alpha, \beta \in \mathbb{C}$, with $v\left(S_{\lambda}\right)=1$. If

$$
z^{-\alpha} \bar{z}^{-\beta} f(-1 / z)=v(T) f(z)+q(z)
$$

for all $z \in \mathcal{H}$, where

$$
q(i y)=\sum_{j=1}^{J}(i y)^{\alpha_{j}} \sum_{t=0}^{T} \beta_{j, t}[\log (i y)]^{t}, \quad y>0,
$$

we call $f$ a nonanalytic automorphic integral of coweights $\alpha, \beta$ and multiplier system $v$ on $\mathcal{G}_{\lambda}$. The function $q(z)$ is called an axial log-polynomial sum.

This reduces to the previous definition when $M=1, \omega_{1}=0, \beta=0$, $\alpha=k \in \mathbb{R}$, and $a_{n_{1}, n_{2}, m}=0$ for $n_{2} \neq 0$. In fact, we will see in Section 5 that if $f$ is analytic, $\beta$ must be zero.

REMARK 2.1. The case $\alpha=-\beta \in \mathbb{Z},\left\{\omega_{m}\right\} \subseteq \mathbb{Z}, a_{n_{1}, n_{2}, m}$ supported only when $n_{1}$ or $n_{2}=0$ appears in [Kn94], where a direct Hecke theorem was obtained for such functions. There, a smaller class of period functions was allowed.

REMARK 2.2. If $\lambda=1$ in either of the preceding two definitions, replace automorphic by modular; if $q(z)=0$ for all $z \in \mathcal{H}$, replace integral with form. There are several excellent contemporary expositions on the theory of forms [Kn93, Le64, Le66, Ap90, Gu63, Ra77, Sc74, Iw97]. 
REMARK 2.3. Definition 2.2 is motivated in part by certain differential operators to be introduced in the next section. In short, if $f$ is an analytic integral and $\eta$ is such an operator (appropriately chosen), then $\eta f$ is a nonanalytic integral. Also, the class of nonanalytic integrals is itself closed under the application of these operators. Aside from this invariance, the definition is also natural from the point of view of the Hecke correspondence [Pa99]. Finally, our notion of nonanalytic integral encompasses such oftstudied examples as the nonanalytic Eisenstein series which we will describe in Section 4.1.

REMARK 2.4. It might seem that we should define an intermediate class of functions, midway between the analytic and nonanalytic automorphic integrals. Namely, insist on the shape of an analytic integral (exponential series) but allow complex first coweight, nonzero second coweight and an axial log-polynomial period function. However, we shall show in Theorem 5.1 that this simply results in Definition 2.1 again, albeit with complex weight. In particular, we will show that if

$$
f(z)=\sum_{n=0}^{\infty} a_{n} e^{2 \pi i n z / \lambda}
$$

and

$$
z^{-\alpha} \bar{z}^{-\beta} f(-1 / z)=C f(z)+q(z),
$$

where $q$ is an axial log-polynomial sum, then either $f$ is constant or $\beta=0$. In either case, then, $f$ is simply an "automorphic integral of complex weight".

3. Weight-changing operators. This study of nonanalytic automorphic integrals is motivated in large part by properties of functions obtained when one applies certain linear differential operators to analytic integrals. Let us survey these operators briefly.

We begin with the well-known weight-raising operator

$$
\delta_{k}=\frac{d}{d z}+\frac{k}{2 i y}, \quad k \in \mathbb{Z} .
$$

If $f$ is an (analytic) modular integral of weight $k$ and identity multiplier system with rational period function $q$, then $\delta_{k} f$ is a nonanalytic modular integral of weight $k+2$ with axial rational period function, that is, a realanalytic function of $z$ and $\bar{z}$ whose restriction to the imaginary axis is a rational function of $z=i y$. To be more precise, the period function of $\delta_{k} f$ is $\delta_{k} q$. Also, the Mellin transforms of $f$ and $\delta_{k} f$ are closely related [Kn83].

In fact, the restrictions on weight, group, multiplier system and analyticity are unnecessary. Accordingly, for $\alpha, \beta \in \mathbb{C}$ we define the first coweight- 
raising operator:

$$
\delta_{\alpha, \beta}=\frac{\partial}{\partial z}+\frac{\alpha}{2 i y} .
$$

Observe that if $f$ is a nonanalytic automorphic integral with axial logpolynomial period function $q$ on $\mathcal{G}_{\lambda}$ of coweights $\alpha, \beta$ and multiplier system $v$, then $\delta_{\alpha, \beta} f$ is a nonanalytic automorphic integral of coweights $\alpha+2, \beta$ and multiplier system $v$ with axial log-polynomial period function $\delta_{\alpha, \beta} q$ on $\mathcal{G}_{\lambda}$.

There is also a weight-lowering operator:

$$
\partial_{k}=y^{2} \frac{\partial}{\partial \bar{z}}
$$

which (together with $\delta_{k}$ ) is applied to nonanalytic forms in [Fr85]. Unlike $\delta_{k}$, however, $\partial_{k}$ does not work on nonanalytic integrals if the second coweight is nonzero. To adapt $\partial_{k}$ to the present circumstances we will put $\partial_{\alpha, \beta}=y^{2} \frac{\partial}{\partial \bar{z}}+$ $\frac{\beta i y}{2}$. (Compare this with the definition of $\delta_{\alpha, \beta}$.) $\partial_{\alpha, \beta}$ lowers the first coweight by 2 ; if $f$ is a nonanalytic automorphic integral with axial log-polynomial period function $q$ on $\mathcal{G}_{\lambda}$ of coweights $\alpha, \beta$ and multiplier system $v$, then $\partial_{\alpha, \beta} f$ is a nonanalytic automorphic integral with axial log-polynomial period function $\partial_{\alpha, \beta} q$ on $\mathcal{G}_{\lambda}$ of coweights $\alpha-2, \beta$ and multiplier system $v$.

Both $\delta_{k}$ and $\partial_{k}$ can be traced back ultimately to Maass's classic work [Ma64]. The operators which will be useful for our purposes are summarized in Table 1. Proofs of the various rules stated therein follow from straightforward calculations based on

$$
\frac{\partial}{\partial z}=\frac{1}{2}\left(\frac{\partial}{\partial x}-i \frac{\partial}{\partial y}\right) \quad \text { and } \quad \frac{\partial}{\partial \bar{z}}=\frac{1}{2}\left(\frac{\partial}{\partial x}+i \frac{\partial}{\partial y}\right),
$$

together with the binary argument convention and the consistency condition. As usual, $y=\operatorname{Im} z$.

\section{Table 1}

\begin{tabular}{ccccc}
\hline Operator & Definition & $\alpha, \beta \rightarrow$ & $v \rightarrow$ & If $f$ is analytic, $\Phi_{f} \rightarrow$ \\
\hline$\delta_{\alpha, \beta}$ & $\frac{\partial}{\partial z}+\frac{\alpha}{2 i y}$ & $\alpha+2, \beta$ & $v$ & $p(s+\alpha) \Phi_{f}(s-1)$ \\
$\partial_{\alpha, \beta}$ & $y^{2} \frac{\partial}{\partial \bar{z}}+\frac{\beta i y}{2}$ & $\alpha-2, \beta$ & $v$ & $\frac{\beta i}{2} \Phi_{f}(s+1)$ \\
$\widetilde{\delta}_{\alpha, \beta}$ & $\frac{\partial}{\partial \bar{z}}-\frac{\beta}{2 i y}$ & $\alpha, \beta+2$ & $v$ & $\frac{\beta i}{2} \Phi_{f}(s-1)$ \\
$\widetilde{\partial}_{\alpha, \beta}$ & $y^{2} \frac{\partial}{\partial z}-\frac{\alpha i y}{2}$ & $\alpha, \beta-2$ & $v$ & $p(s+1) \Phi_{f}(s+1)$ \\
$S_{w}$ & $y^{-w}$ & $\alpha+w, \beta+w$ & $v$ & $\Phi_{f}(s+w)$ \\
$\mathrm{C}$ & $f \rightarrow \bar{f}$ & $\bar{\beta}, \bar{\alpha}$ & $\bar{v}$ & $\overline{\Phi_{f}(\bar{s})}$ \\
$\mathrm{C}$ & $z \rightarrow-\bar{z}$ & $\beta, \alpha$ & $v_{1}$ & $\Phi_{f}(s)$ \\
\hline
\end{tabular}

Here, $\alpha, \beta, w$ are arbitrary complex numbers; $p(s)=i(s-1-\alpha / 2)$; $v_{1}=v \circ \varsigma$, where $\varsigma$ is the involution which negates the off-diagonal elements 
of $M=\left[\begin{array}{ll}a & b \\ c & d\end{array}\right] ; \Phi_{g}$ denotes the Mellin transform of $g$ :

$$
\Phi_{g}(s)=\int_{0}^{\infty}\left[g(i y)-\sum_{m=1}^{M} a_{0,0, m} y^{\omega_{m}}\right] y^{s-1} d y, \quad \text { Re } s \text { large, }
$$

where $g(z)=\sum_{m=1}^{M} y^{\omega_{m}} \sum_{n_{1}, n_{2}=0}^{\infty} a_{n_{1}, n_{2}, m} \exp \left[2 \pi i \lambda^{-1}\left(n_{1} z-n_{2} \bar{z}\right)\right]$. (The connection between the Mellin transforms of these functions, which are summarized in the last column of the table, has several applications. Among these: a Hecke correspondence for analytic integrals with a restricted set of rational period functions [Kn83], and a growth estimate on the Mellin transforms of analytic integrals [Pa99].) It is important to interpret exponents according to the binary argument convention here, in order that we have

$$
|c z+d|^{2 w}=(c z+d)^{w}(c \bar{z}+d)^{w}
$$

for all $z \in \mathcal{H}, w \in \mathbb{C}$ and $c, d \in \mathbb{R}$ such that $|c|+|d| \neq 0$.

Note that the first five operators in Table 1 have infinite order, while Ç and ç have order 2. Also, these five operators preserve both $v$ and the set $\alpha-\beta+2 \mathbb{Z}$. This is no coincidence, in light of the remarks preceding Definition 2.1 ; for the multiplier system to be preserved, the coweight-difference can change only by an even integer.

Analyticity is not, in general, preserved by these operators, the only nontrivial exception being the composition $\mathrm{C} \cdot c ̧$.

As far as we know, $\partial_{\alpha, \beta}, \widetilde{\partial}_{\alpha, \beta}, \widetilde{\delta}_{\alpha, \beta}$ and $S_{w}$ appear here for the first time, except for $S_{-k}, k \in \mathbb{Z}$, which was applied to analytic integrals of weight $2 k$ to construct nonanalytic integrals of coweights $k,-k$ and identity multiplier system in [Kn94]. Ç, ç have been used previously with nonanalytic integrals of real coweights $\alpha, \beta$, with $\beta=-\alpha$ [ibid.], in which case each operator preserves both coweights; and $C$ has been applied to forms with real coweights in [Ma64].

Each operator in Table 1 preserves the shape of axial log-polynomial sums, and therefore the coweights of a nonanalytic automorphic integral change as described in the third column of the table.

It would appear that, armed with Table 1, we might generate an infinite supply of nonanalytic examples by applying $\delta$, $\partial$, etc. to analytic integrals; however, it is appropriate to note first the limitations of such an approach, because of certain algebraic relations. For example, if $f$ is an (analytic) integral of coweights $\alpha, \beta$ and multiplier system $v$ on $\mathcal{G}_{\lambda}$, then apparently we have (at least) two constructions of a nonanalytic integral of coweights $\alpha+2, \beta: \delta_{\alpha, \beta} f$ and $S_{\alpha+2} \widetilde{\partial}_{0, \beta-\alpha} S_{-\alpha} f$. Unfortunately, both constructions result in the same function; $\delta_{\alpha, \beta}=S_{\alpha+2} \widetilde{\partial}_{0, \beta-\alpha} S_{-\alpha}$. More generally, nontrivial relations among the operators are summarized below. 
Table 2

\begin{tabular}{ll}
\hline \multicolumn{1}{c}{ Relation } & $\alpha, \beta \rightarrow$ \\
\hline$\partial_{\alpha, \beta}=S_{\alpha-2} \widetilde{\delta}_{0, \beta-\alpha} S_{-\alpha}$ & $\alpha-2, \beta$ \\
$\widetilde{\partial}_{\alpha, \beta}=S_{\beta-2} \delta_{\alpha-\beta, 0} S_{-\beta}$ & $\alpha, \beta-2$ \\
$\delta_{\alpha, \beta}=S_{\alpha+2} \widetilde{\partial}_{0, \beta-\alpha} S_{-\alpha}$ & $\alpha+2, \beta$ \\
$\widetilde{\delta}_{\alpha, \beta}=S_{\beta+2} \partial_{\alpha-\beta, 0} S_{-\beta}$ & $\alpha, \beta+2$ \\
$\delta_{\alpha, \beta-2} \widetilde{\partial}_{\alpha, \beta}=\widetilde{\partial}_{\alpha+2, \beta} \delta_{\alpha, \beta}$ & $\alpha+2, \beta-2$ \\
$\widetilde{\delta}_{\alpha-2, \beta} \partial_{\alpha, \beta}=\partial_{\alpha, \beta+2} \widetilde{\delta}_{\alpha, \beta}$ & $\alpha-2, \beta+2$ \\
$\delta_{\alpha-2, \beta} \partial_{\alpha, \beta}=\partial_{\alpha+2, \beta} \delta_{\alpha, \beta}+(\alpha-\beta) / 4$ & $\alpha, \beta$ \\
$\widetilde{\partial}_{\alpha, \beta+2} \widetilde{\delta}_{\alpha, \beta}=\widetilde{\delta}_{\alpha, \beta-2} \widetilde{\partial}_{\alpha, \beta}+(\alpha-\beta) / 4$ & $\alpha, \beta$ \\
$\delta_{\alpha, \beta+2} \widetilde{\delta}_{\alpha, \beta}=\widetilde{\delta}_{\alpha+2, \beta} \delta_{\alpha, \beta}+(\alpha-\beta) S_{2} / 4$ & $\alpha+2, \beta+2$ \\
$\widetilde{\partial}_{\alpha-2, \beta} \partial_{\alpha, \beta}=\partial_{\alpha, \beta-2} \widetilde{\partial}_{\alpha, \beta}+(\alpha-\beta) S_{-2} / 4$ & $\alpha-2, \beta-2$ \\
$\delta_{\alpha, \beta}=S_{\gamma+2} \widetilde{\partial}_{\alpha-\gamma, \varepsilon} S_{-\gamma}$ & $\alpha+2, \beta$ \\
$\widetilde{\delta}_{\alpha, \beta}=S_{\gamma+2} \partial_{\varepsilon, \beta+\gamma} S_{-\gamma}$ & $\alpha, \beta+2$ \\
\hline
\end{tabular}

$(\alpha, \beta, \gamma, \varepsilon$ are arbitrary complex numbers.) Fortunately, as we will show in the next section, these relations do not impose a significant limitation on our ability to generate a large and interesting class of examples.

\section{Examples}

4.1. Analytic integrals, weight-changing operators and Eisenstein series. The space of nonanalytic automorphic integrals contains the subspace of analytic integrals, so immediately we have a wealth of examples to draw on which have been much-discussed in the literature [Ei57, Kn83, Kn89a]. From these, one obtains many more examples by application of the coweightchanging operators described in the previous section, and still further by taking certain linear combinations or (in the case of forms) products of these.

Another example is the nonanalytic Eisenstein series of integer coweights, defined by

$$
G_{\alpha, \beta}(z)=\sum_{c, d \in \mathbb{Z}}^{\prime}(c z+d)^{-\alpha}(c \bar{z}+d)^{-\beta},
$$

where $\alpha, \beta \in \mathbb{Z}, \alpha+\beta>2, \sum^{\prime}$ excludes the term $(c, d)=(0,0)$ and we interpret $c=0$ terms according to the binary argument convention. This is a nonanalytic modular form of coweights $\alpha, \beta$ and identity multiplier system 
[Ma64]. (Note that when $\alpha+\beta=2$, we may write a conditionally convergent Eisenstein series which is a modular integral, unless $\alpha=\beta=1$, in which case the series is divergent for any particular ordering [Hu81]. In particular, Hurwitz's work demonstrates that

$$
G_{2,0}(z)=\sum_{c}\left\{\sum_{d}^{\prime}(c z+d)^{-2}\right\}
$$

has identity multiplier system, coweights 2,0 and period function $-2 \pi i / z$. It follows that

$$
G_{0,2}(z)=\sum_{c}\left\{\sum_{d}^{\prime}(c \bar{z}+d)^{-2}\right\}
$$

is conditionally convergent as well, and has identity multiplier system, coweights 0,2 and period function $2 \pi i / \bar{z}$. It also follows that

diverges.)

$$
G_{1,1}(z)=\sum_{c}\left\{\sum_{d}^{\prime}|c \bar{z}+d|^{-2}\right\}
$$

4.2. Dimensionality. Most treatments of modular or automorphic forms begin with the analytic Eisenstein series or its cousin, the Poincaré series. Unfortunately, this approach fails when one allows arbitrary complex coweights, since there are convergence problems when $\alpha-\beta \notin \mathbb{R}$ [Pa98]. Instead we use a different approach which utilizes the discriminant function $\Delta(z)$, a modular form of weight 12 and identity multiplier system, defined by

$$
\Delta(z)=e^{2 \pi i z} \prod_{n=1}^{\infty}\left(1-e^{2 \pi i n z}\right)^{24}, \quad \operatorname{Im} z>0 .
$$

In the classical theory of (analytic) modular forms, one can show that if $k \in 2 \mathbb{Z}^{+}$, then the space of entire forms of coweights $k, 0$ and identity multiplier system on the full modular group has dimension

$$
d_{k}= \begin{cases}\lfloor k / 12\rfloor & \text { if } k=2(\bmod 12), \\ \lfloor k / 12\rfloor+1 & \text { if } k \neq 2(\bmod 12)\end{cases}
$$

(see [Ser70, Ap90]; $\lfloor x\rfloor$ denotes the greatest integer less than or equal to $x$ ). In fact, for arbitrary real weight the space of entire forms is finite-dimensional and the dimension is known [Le64].

In stark contrast to this cozy scene, the space of nonanalytic modular forms for these same coweights and multiplier system is infinite-dimensional. We will prove this in an even more general situation, by way of an explicit construction which uses the weight-changing operators $\partial, \widetilde{\partial}$, and $C$.

Let $M^{+}\{\lambda, \alpha, \beta, v\}$ be the space of nonanalytic automorphic forms on $\mathcal{G}_{\lambda}$ of coweights $\alpha, \beta \in \mathbb{C}$ and multiplier system $v$, and let $M^{0}\{\lambda, \alpha, \beta, v\}$ be 
the subspace of forms which vanish as $z \rightarrow i \infty$ in $|\operatorname{Re} z| \leq T$, with $T>0$ fixed. (In the analytic case, these are called cusp forms.)

Theorem 4.1. If $M^{+}\{1, \alpha, \beta, v\} \neq\{0\}$, then $\operatorname{dim}_{\mathbb{C}} M^{0}\{1, \alpha, \beta, v\}=\infty$.

The theorem follows from the next lemma.

Lemma 4.2. For $l \in \mathbb{Z}^{+} \cup\{0\}$, put $h_{l}(z)=\widetilde{\partial}^{6} \partial^{l+2}\left[G_{2 l+4,0}(z)(C \zeta \Delta)(z)\right]$. $A$ linearly independent set of nonanalytic modular forms of coweights 0,0 and identity multiplier system is given by $\left\{h_{l}(z)\right\}_{l=0}^{\infty}$. Moreover, $h_{l}(z) \rightarrow 0$ as $z \rightarrow i \infty,|\operatorname{Re} z| \leq T$.

Proof. For $l \in \mathbb{Z}^{+} \cup\{0\}, G_{2 l+4,0}(z) \overline{\Delta(z)}$ is a nonanalytic modular form of coweights $2 l+4,12$ and multiplier system $v \equiv 1$. By Table 1 , then, $h_{l}(z)$ is a nonanalytic form of coweights 0,0 .

In the present context,

$$
\widetilde{\partial}^{6}=\widetilde{\partial}_{0,2} \cdot \widetilde{\partial}_{0,4} \cdot \ldots \cdot \widetilde{\partial}_{0,12}=\left(y^{2} \frac{\partial}{\partial z}\right)^{6}=y^{12} \frac{\partial^{6}}{\partial z^{6}}+\sum_{j=1}^{5} c_{j} y^{6+j} \frac{\partial^{j}}{\partial z^{j}}
$$

and

$$
\begin{aligned}
\partial^{l+2} & =\partial_{2,12} \cdot \partial_{4,12} \cdot \ldots \cdot \partial_{2 l+2,12} \cdot \partial_{2 l+4,12}=\left(y^{2} \frac{\partial}{\partial \bar{z}}+6 i y\right)^{l+2} \\
& =y^{2 l+4} \frac{\partial^{l+2}}{\partial \bar{z}^{l+2}}+\sum_{m=1}^{2 l+3} y^{m} \sum_{j=1}^{l+1} \varepsilon_{l, m, j} \frac{\partial^{j}}{\partial \bar{z}^{j}}+p_{l+2}(y)
\end{aligned}
$$

$\left(c_{j}, \varepsilon_{l, m, j}\right.$ are complex constants, and $p_{l+2}$ is a polynomial of degree $\leq l+2$.) Also, for $z \in \mathcal{H}$,

$$
G_{2 l+4,0}(z)=2 \zeta(2 l+4)+\frac{2 \cdot(2 \pi)^{2 l+4}(-1)^{l}}{(2 l+3) !} \sum_{n=1}^{\infty} \sigma_{2 l+3}(n) e^{2 \pi i n z},
$$

where

$$
\sigma_{k}(n)=\sum_{d \mid n, d>0} d^{k} \quad \text { and } \quad \zeta(b)=\sum_{m=1}^{\infty} m^{-b}
$$

[Ap90]. Finally, by definition of the Ramanujan $\tau$-function, we have

$$
\Delta(z)=\sum_{n=1}^{\infty} \tau(n) e^{2 \pi i n z}, \quad z \in \mathcal{H}
$$

Hence, 


$$
\begin{aligned}
\partial^{l+2}\left[G_{2 l+4,0}(z)(\mathrm{C} \Delta)(z)\right] & =G_{2 l+4,0}(z) \partial^{l+2}[(\mathrm{C} \Delta)(z)] \\
= & G_{2 l+4,0}(z)\left[y^{2 l+4} \frac{\partial^{l+2}}{\partial \bar{z}^{l+2}}+\sum_{m=1}^{2 l+3} y^{m} \sum_{j=1}^{l+1} \varepsilon_{l, m, j} \frac{\partial^{j}}{\partial \bar{z}^{j}}+p_{l+2}(y)\right][\overline{\Delta(z)}] \\
= & G_{2 l+4,0}(z)\left(y^{2 l+4} \frac{\partial^{l+2}}{\partial \bar{z}^{l+2}}+\sum_{m=1}^{2 l+3} y^{m} \sum_{j=1}^{l+1} \varepsilon_{l, m, j} \frac{\partial^{j}}{\partial \bar{z}^{j}}\right) \overline{\Delta(z)} \\
& +p_{l+2}(y) G_{2 l+4,0}(z) \overline{\Delta(z)} \\
= & G_{2 l+4,0}(z)\left(y^{2 l+4} \frac{\partial^{l+2}}{\partial \bar{z}^{l+2}}+\sum_{m=1}^{2 l+3} y^{m} \sum_{j=1}^{l+1} \varepsilon_{l, m, j} \frac{\partial^{j}}{\partial \bar{z}^{j}}\right) \sum_{n=1}^{\infty} \tau(n) e^{-2 \pi i n \bar{z}} \\
& +p_{l+2}(y) G_{2 l+4,0}(z) \overline{\Delta(z) .}
\end{aligned}
$$

Thus,

$$
\begin{aligned}
\partial^{l+2}\left[G_{2 l+4,0}(z)(\mathrm{C} \Delta)(z)\right] & \\
= & G_{2 l+4,0}(z)\left[y^{2 l+4} \sum_{n=1}^{\infty}(-2 \pi i n)^{l+2} \tau(n) e^{-2 \pi i n \bar{z}}\right. \\
& \left.+\sum_{m=1}^{2 l+3} y^{m} \sum_{j=1}^{l+1} \varepsilon_{l, m, j} \sum_{n=1}^{\infty}(-2 \pi i n)^{j} \tau(n) e^{-2 \pi i n \bar{z}}\right] \\
& +p_{l+2}(y) G_{2 l+4,0}(z) \overline{\Delta(z)} .
\end{aligned}
$$

It follows, then, that

$$
\begin{aligned}
h_{l}(z)= & \widetilde{\partial}^{6} \partial^{l+2}\left[G_{2 l+4,0}(z)(\mathrm{C} \Delta)(z)\right] \\
= & \widetilde{\partial}^{6}\left[G_{2 l+4,0}(z) y^{2 l+4} \sum_{n=1}^{\infty}(-2 \pi i n)^{l+2} \tau(n) e^{-2 \pi i n \bar{z}}\right] \\
& +\widetilde{\partial}^{6}\left[G_{2 l+4,0}(z) \sum_{m=1}^{2 l+3} y^{m} \sum_{j=1}^{l+1} \varepsilon_{l, m, j} \sum_{n=1}^{\infty}(-2 \pi i n)^{j} \tau(n) e^{-2 \pi i n \bar{z}}\right] \\
& +\widetilde{\partial}^{6}\left[p_{l+2}(y) G_{2 l+4,0}(z) \overline{\Delta(z)}\right] \\
= & \sum_{n=1}^{\infty}(-2 \pi i n)^{l+2} \tau(n) e^{-2 \pi i n \bar{z}} \widetilde{\partial}^{6}\left[y^{2 l+4} G_{2 l+4,0}(z)\right] \\
& +\sum_{n=1}^{\infty}(-2 \pi i n)^{j} \tau(n) e^{-2 \pi i n \bar{z}} \widetilde{\partial}^{6}\left[G_{2 l+4,0}(z) \sum_{m=1}^{2 l+3} y^{m} \sum_{j=1}^{l+1} \varepsilon_{l, m, j}\right] \\
& +\overline{\Delta(z)} \widetilde{\partial}^{6}\left[p_{l+2}(y) G_{2 l+4,0}(z)\right] .
\end{aligned}
$$


Therefore,

$$
\begin{aligned}
& h_{l}(z)=\sum_{n=1}^{\infty}(-2 \pi i n)^{l+2} \tau(n) e^{-2 \pi i n \bar{z}}\left(y^{12} \frac{\partial^{6}}{\partial z^{6}}+\sum_{j=1}^{5} c_{j} y^{6+j} \frac{\partial^{j}}{\partial z^{j}}\right) \\
& \times\left[y^{2 l+4} G_{2 l+4,0}(z)\right] \\
& +\sum_{n=1}^{\infty}(-2 \pi i n)^{j} \tau(n) e^{-2 \pi i n \bar{z}}\left(y^{12} \frac{\partial^{6}}{\partial z^{6}}+\sum_{j=1}^{5} c_{j} y^{6+j} \frac{\partial^{j}}{\partial z^{j}}\right) \\
& \times G_{2 l+4,0}(z) \sum_{m=1}^{2 l+3} y^{m} \sum_{j=1}^{l+1} \varepsilon_{l, m, j} \\
& +\overline{\Delta(z)}\left(y^{12} \frac{\partial^{6}}{\partial z^{6}}+\sum_{j=1}^{5} c_{j} y^{6+j} \frac{\partial^{j}}{\partial z^{j}}\right)\left[p_{l+2}(y) G_{2 l+4,0}(z)\right] \\
& =y^{2 l+16}\left[\frac{\partial^{6}}{\partial z^{6}} G_{2 l+4,0}(z)\right] \sum_{n=1}^{\infty}(-2 \pi i n)^{l+2} \tau(n) e^{-2 \pi i n \bar{z}} \\
& +\sum_{m=1}^{2 l+15} y^{m} \sum_{n_{1}, n_{2}=0}^{\infty} a_{n_{1}, n_{2}, m}(l) e^{2 \pi i\left(n_{1}-n_{2}\right) z} \\
& =y^{2 l+16}\left[\frac{2 \cdot(2 \pi)^{2 l+4}(-1)^{l}}{(2 l+3) !} \sum_{n=1}^{\infty}(2 \pi i n)^{6} \sigma_{2 l+3}(n) e^{2 \pi i n z}\right] \\
& \times \sum_{n=1}^{\infty}(-2 \pi i n)^{l+2} \tau(n) e^{-2 \pi i n \bar{z}} \\
& +\sum_{m=1}^{2 l+15} y^{m} \sum_{n_{1}, n_{2}=0}^{\infty} a_{n_{1}, n_{2}, m}(l) e^{2 \pi i\left(n_{1}-n_{2}\right) z} .
\end{aligned}
$$

Therefore, for $y>0$,

$$
\begin{aligned}
h_{l}(i y)= & \frac{2^{3 l+12} \pi^{3 l+12} i^{l}}{(2 l+3) !} y^{2 l+16} \sum_{n_{1}, n_{2}=1}^{\infty} n_{1}^{6} n_{2}^{l+2} \sigma_{2 l+3}\left(n_{1}\right) \tau\left(n_{2}\right) e^{-2 \pi\left(n_{1}+n_{2}\right) y} \\
& +\sum_{m=1}^{2 l+15} y^{m} \sum_{n=0}^{\infty} c_{n, m}(l) e^{-2 \pi n y} .
\end{aligned}
$$

Thus, the coefficient of $y^{2 l+16} e^{-2 \pi(2 y)}$ in the expansion of $h_{l}(i y)$ is

$$
\frac{(-2 \pi i)^{3 l+12}}{(2 l+3) !} \neq 0
$$

while the coefficient of $y^{2 l+16} e^{-2 \pi(2 y)}$ in the expansion of $h_{l_{0}}(i y)$ is zero for all 
$l_{0}<l$. By the uniqueness of the representation $\sum_{m=1}^{M} y^{m} \sum_{n=0}^{\infty} d_{n, m} e^{-2 \pi n y}$ (which is easily checked!), this establishes the linear independence of the $h_{l}$.

It remains to be shown that $h_{l} \rightarrow 0$ as $z \rightarrow i \infty,|\operatorname{Re} z| \leq T$. But we have already seen that

$$
h_{l}(z)=e^{-2 \pi i \bar{z}} \sum_{m=1}^{2 l+16} y^{m} \sum_{n_{1}, n_{2}=0}^{\infty} a_{n_{1}, n_{2}, m}(l) e^{2 \pi i\left(n_{1} z-n_{2} \bar{z}\right)}
$$

for $z \in \mathcal{H}$. Since $e^{-2 \pi i \bar{z}} \rightarrow 0$ and the rest of the expression approaches

$$
\sum_{m=1}^{2 l+16} y^{m} a_{0,0, m}(l)
$$

the desired limit holds. (Note that $a_{n_{1}, n_{2}, m}(l)$ satisfies the usual growth condition $\sum_{n_{1}+n_{2}=n}\left|a_{n_{1}, n_{2}, m}(l)\right|=\mathcal{O}\left(n^{\gamma}\right)$, because these coefficients are finite sums of products of $\sigma_{2 l+3}\left(n_{1}\right)$ and $\tau\left(n_{2}\right)$, each of which exhibits polynomial growth.)

Theorem 4.1 follows because if there exists a nonzero nonanalytic modular form $f$ on $\mathcal{G}_{\lambda}$ of coweights $\alpha, \beta$ and multiplier system $v$, then $f(z) h_{l}(z)$ is a nonanalytic modular form associated with the same group, coweights and multiplier system. (More generally, if $f_{1} \in M^{+}\left\{\lambda, \alpha_{1}, \beta_{1}, v_{1}\right\}$ and $f_{2} \in$ $M^{+}\left\{\lambda, \alpha_{2}, \beta_{2}, v_{2}\right\}$, then $f_{1} f_{2} \in M^{+}\left\{\lambda, \alpha_{1}+\alpha_{2}, \beta_{1}+\beta_{2}, v_{1} v_{2}\right\}$, a fact we have used implicitly already.)

Corollary 4.3. For $k \in 2 \mathbb{Z}^{+}, k>2, \operatorname{dim}_{\mathbb{C}} M^{0}\{1, k, 0,1\}=\infty$.

Proof. $G_{k, 0} \in M^{+}\{1, k, 0,1\}$.

4.3. A construction of nonanalytic forms for arbitrary complex coweights. Next we will give an explicit construction, for given complex coweights, of nonanalytic forms on the theta group. First, suppose that $\operatorname{Re} \alpha, \operatorname{Re} \beta>0$. The classical theta function

$$
\vartheta(z)=\sum_{n=-\infty}^{\infty} e^{\pi i n^{2} z}
$$

$z \in \mathcal{H}$, is a modular form of weight $1 / 2$ on $\mathcal{G}_{2}$, with $\vartheta(-1 / z)=\sqrt{-i z} \vartheta(z)$. $\vartheta$ has the infinite product representation

$$
\vartheta(z)=\prod_{n=1}^{\infty}\left(1-e^{2 n \pi i z}\right)\left(1+e^{(2 n-1) \pi i z}\right)^{2},
$$

$z \in \mathcal{H}$ [Kn93]. It follows that $\vartheta$ is nonvanishing in $\mathcal{H}$; ergo $\log \vartheta$ is a singlevalued analytic function. Since $\vartheta(-1 / z)=\sqrt{-i z} \vartheta(z)=e^{-i \pi / 4} \sqrt{z} \vartheta(z)$,

$$
\log \vartheta(-1 / z)=-i \pi / 4+\frac{1}{2} \log z+\log \vartheta(z)+2 \pi i A \quad \text { for some } A \in \mathbb{Z} .
$$


Putting $z=i$, we get $A=0$. Thus we have

$$
2 \alpha \log \vartheta(-1 / z)=-i \pi \alpha / 2+\alpha \log z+2 \alpha \log \vartheta(z) .
$$

Exponentiation gives $\vartheta^{2 \alpha}(-1 / z)=e^{-i \pi \alpha / 2} z^{\alpha} \vartheta^{2 \alpha}(z)$.

On the other hand, for $y>0$,

$$
\begin{aligned}
\vartheta^{2 \alpha}(i y) & =\left[\prod_{n=1}^{\infty}\left(1-e^{-2 n \pi y}\right)\left(1+e^{-(2 n-1) \pi y}\right)^{2}\right]^{2 \alpha} \\
& =\prod_{n=1}^{\infty}\left(1-e^{-2 n \pi y}\right)^{2 \alpha}\left(1+e^{-(2 n-1) \pi y}\right)^{4 \alpha}
\end{aligned}
$$

and so by the identity theorem

$$
\vartheta^{2 \alpha}(z)=\prod_{n=1}^{\infty}\left(1-e^{2 n \pi i z}\right)^{2 \alpha}\left(1+e^{(2 n-1) \pi i z}\right)^{4 \alpha} .
$$

Thus $\vartheta^{2 \alpha}(z+2)=\vartheta^{2 \alpha}(z)$, and we may conclude that $\vartheta^{2 \alpha}$ is an analytic form of coweights $\alpha, 0$ on $\mathcal{G}_{2}$ with multiplier system $v_{1}$ generated by $v_{1}\left(S_{2}\right)=1$, $v_{1}(T)=e^{-i \pi \alpha / 2}$ and the consistency condition. (For a discussion of the Fourier coefficients of $\vartheta^{2 \alpha}$ when $\alpha$ is real, see [Ma38, Si56, Kn86, Kn89].)

By Table 1, then, $\overline{\vartheta^{2 \bar{\beta}}}$ has coweights $0, \beta$ and multiplier system $v_{2}$ generated by $v_{1}\left(S_{2}\right)=1, v_{1}(T)=e^{i \pi \beta / 2}$. If we put $f(z)=\vartheta^{2 \alpha}(z) \overline{\vartheta^{2 \bar{\beta}}(z)}$, then $f$ is a nonanalytic form on the theta group of coweights $\alpha, \beta$ and multiplier system $v_{\alpha, \beta}$ generated by $v_{\alpha, \beta}\left(S_{2}\right)=1, v_{\alpha, \beta}(T)=e^{i \pi(\beta-\alpha) / 2}$. Thus by Theorem 4.1, $\operatorname{dim}_{\mathbb{C}} M^{0}\left\{2, \alpha, \beta, v_{\alpha, \beta}\right\}=\infty$.

To relax the assumptions on $\operatorname{Re} \alpha$ and $\operatorname{Re} \beta$, merely apply the weightlowering operators $\partial, \widetilde{\partial}$.

5. A theorem. Now we may prove Remark 2.4, which states that our definition of nonanalytic integral is in some sense the most economical one which both encompasses known examples and is closed under the coweightchanging operators.

Recall that the generalization from analytic to nonanalytic integrals considered here is threefold: allow a second coweight, admit a broader class of period functions, and give $f$ a more general shape than simple exponential series. The next theorem shows that the last of these is necessary to make our concept of nonanalytic integral a meaningful one.

Theorem 5.1. For $z \in \mathcal{H}$, define

$$
f(z)=\sum_{n=0}^{\infty} a_{n} e^{2 \pi i n z / \lambda},
$$


where $a_{n}=O\left(n^{\gamma}\right), \gamma>0$, as $n \rightarrow \infty$. Let $v$ be a multiplier system on $\mathcal{G}_{\lambda}$ of coweights $\alpha, \beta \in \mathbb{C}$, with $v\left(S_{\lambda}\right)=1$. If

$$
z^{-\alpha} \bar{z}^{-\beta} f\left(\frac{-1}{z}\right)=v(T) f(z)+q(z) \quad \text { for } z \in \mathcal{H},
$$

where $q$ is an axial log-polynomial sum, then either $\beta=0$ or $f$ is constant.

REMARK 5.1. The theorem holds even if $f$ has the more general form

$$
f(z)=\sum_{n=0}^{\infty} a_{n} e^{2 \pi i(n+\kappa) z / \lambda}
$$

$0 \leq \operatorname{Re} \kappa<1$, and our proof easily extends to this case. Our principal tools in the proof are several operators from Table 1 and the next lemma:

Lemma 5.2. Let $\theta>0$. A log-polynomial sum which decays exponentially as $z \rightarrow \infty$ within a set of the form $W_{\theta}=\{z \in \mathbb{C}: z \neq 0,|\pi / 2-\arg z|<\theta\}$ is identically zero (Figure 1).

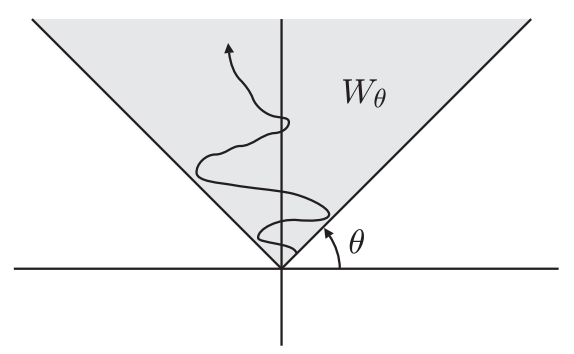

Fig. 1. $z \rightarrow i \infty$ in $W_{\theta}$

Proof. Let $q(z)=\sum_{j=0}^{J} z^{\alpha_{j}} \sum_{t=0}^{T} \beta_{j, t}(\log z)^{t}$, where the $\alpha_{j}$ are distinct complex numbers, ordered lexicographically. That is, $\operatorname{Re} \alpha_{j} \leq \operatorname{Re} \alpha_{j+1}$, and $\operatorname{Im} \alpha_{j}<\operatorname{Im} \alpha_{j+1}$ if $\operatorname{Re} \alpha_{j}=\operatorname{Re} \alpha_{j+1}$ for $j=1, \ldots, J-1$. We will proceed by strong induction on $J$.

The case $J=1$ is not difficult. If $q(z)=z^{\alpha_{1}} \sum_{t=0}^{T} \beta_{1, t}(\log z)^{t} \rightarrow 0$ exponentially as $z \rightarrow \infty$ in $W_{\theta}$, then the same is true of $\sum_{t=0}^{T} \beta_{1, t}(\log z)^{t}$. Put $\omega=\log z$. Then $\sum_{t=0}^{T} \beta_{1, t} \omega^{t} \rightarrow 0$ as $\omega \rightarrow \infty$ in the horizontal strip $\{\omega \in \mathbb{C}: \theta \leq \operatorname{Im} \omega \leq \pi-\theta\}$. But $\sum_{t=0}^{T} \beta_{1, t} \omega^{t}$ is a polynomial, so it is identically zero. Thus $q(z) \equiv 0$.

Next we consider the case $J>1$. It was proved by Hassen in [Ha99] that if $c_{1}, \ldots, c_{N}$ are complex numbers not all zero, $u_{1}, \ldots, u_{N}$ are distinct real numbers and $\lim _{z \rightarrow \infty}$ in $\mathbb{C} \sum_{j=1}^{N} c_{j} z^{i u_{j}}$ exists, then $N=1$ and $u_{1}=0$ (and thus the sum is a constant). In fact, the same proof shows that a stronger result holds; namely, if we assume only that $\lim _{z \rightarrow \infty \text { in } W_{\theta}} \sum_{j=1}^{N} c_{j} z^{i u_{j}}$ exists, then the same conclusion holds. 
Since $\operatorname{Re} \alpha_{j}$ is increasing in $j$, we have $\max _{j} \operatorname{Re} \alpha_{j}=\operatorname{Re} \alpha_{J}$. Say $\operatorname{Re} \alpha_{j}=$ $\operatorname{Re} \alpha_{J}$ iff $j \geq j_{0}$. By assumption, $q(z) \rightarrow 0$ exponentially as $z \rightarrow \infty$ in $W_{\theta}$, so the same is true of $z^{-\alpha_{J}}(\log z)^{-T} q(z)=\sum_{j=1}^{J} z^{\alpha_{j}-\alpha_{J}} \sum_{t=0}^{T} \beta_{j, t}(\log z)^{t-T}$. But $\lim _{z \rightarrow \infty \text { in } W_{\theta}} z^{\alpha_{j}-\alpha_{J}}(\log z)^{t-T}=0$ for $j<j_{0}$. Therefore,

$$
\sum_{j=j_{0}}^{J} z^{\alpha_{j}-\alpha_{J}} \sum_{t=0}^{T} \beta_{j, t}(\log z)^{t-T} \rightarrow 0
$$

Also, $\left|z^{\alpha_{j}-\alpha_{J}}\right|$ is bounded for $j \geq j_{0}$, so

$$
\left|z^{\alpha_{j}-\alpha_{J}} \sum_{t=0}^{T-1} \beta_{j, t}(\log z)^{t-T}\right| \leq\left|z^{\alpha_{j}-\alpha_{J}}\right| \sum_{t=0}^{T-1}\left|\beta_{j, t}\right| \cdot\left|(\log z)^{t-T}\right| \rightarrow 0 .
$$

Thus $\lim _{z \rightarrow \infty \text { in } W_{\theta}} \sum_{j=j_{0}}^{J} z^{\alpha_{j}-\alpha_{J}} \beta_{j, T}=0$. Now we may apply the result of Hassen, with $u_{j}=-i \operatorname{Re}\left(\alpha_{j}-\alpha_{J}\right) \in \mathbb{R}$ and $c_{j}=\beta_{j, T}$, to get $\beta_{j, T}=0$ for all $j \geq j_{0}$.

Thus $q(z)=\sum_{j=1}^{j_{0}-1} z^{\alpha_{j}} \sum_{t=0}^{T} \beta_{j, t}(\log z)^{t}+\sum_{j=j_{0}}^{J} z^{\alpha_{j}} \sum_{t=0}^{T-1} \beta_{j, t}(\log z)^{t}$.

But $z^{-\alpha_{j}}(\log z)^{-(T-1)} q(z)$ still has exponential decay, so as before we can show that $\beta_{j, T-1}=0$ for all $j \geq j_{0}$. Continuing in this fashion, we see that $\beta_{j, t}=0$ for all $j \geq j_{0}, 0 \leq t \leq T$.

Then $q(z)=\sum_{j=1}^{j_{0}-1} z^{\alpha_{j}} \sum_{t=0}^{T} \beta_{j, t}(\log z)^{t}$, where $j_{0}-1<J$. By the induction hypothesis, $\beta_{j, t}=0$ for $1 \leq j \leq j_{0}-1,0 \leq t \leq T$. This completes the proof of the lemma.

Proof of Theorem 5.1. Let $\beta \in \mathbb{C} \backslash\{0\}$. By Table 1 , both $\partial_{\alpha, \beta} f$ and $S_{-1} f$ are nonanalytic automorphic integrals on $\mathcal{G}_{\lambda}$ with multiplier system $v ; \partial_{\alpha, \beta} f$ has coweights $\alpha-2, \beta$ and period function $\partial_{\alpha, \beta} q$, while $S_{-1} f$ has coweights $\alpha-1, \beta-1$ and period function $S_{-1} q$. Since $f$ is analytic, $\partial f / \partial \bar{z}=0$. Thus,

$$
\partial_{\alpha, \beta} f=y^{2} \frac{\partial f}{\partial \bar{z}}+\frac{\beta i y}{2} f=\frac{\beta i y}{2} f=\frac{\beta i}{2} S_{-1} f .
$$

By the transformation laws for $\partial_{\alpha, \beta} f$ and $S_{-1} f$, then, we have

$$
\begin{aligned}
z^{\alpha-1} \bar{z}^{\beta-1}\left[v(T)\left(S_{-1} f\right)(z)\right. & \left.+\left(S_{-1} q\right)(z)\right] \\
& =\left(S_{-1} f\right)\left(\frac{-1}{z}\right)=\frac{2}{\beta i}\left(\partial_{\alpha, \beta} f\right)\left(\frac{-1}{z}\right) \\
& =\frac{2}{\beta i} z^{\alpha-2} \bar{z}^{\beta}\left[v(T)\left(\partial_{\alpha, \beta} f\right)(z)+\left(\partial_{\alpha, \beta} q\right)(z)\right] \\
& =z^{\alpha-2} \bar{z}^{\beta}\left[v(T)\left(S_{-1} f\right)(z)+\frac{2}{\beta i}\left(\partial_{\alpha, \beta} q\right)(z)\right] .
\end{aligned}
$$

Therefore,

$$
\begin{aligned}
\left(z^{\alpha-1} \bar{z}^{\beta-1}-z^{\alpha-2} \bar{z}^{\beta}\right) v(T)\left(S_{-1} f\right)(z) & \\
= & z^{\alpha-2} \bar{z}^{\beta} \frac{2}{\beta i}\left(\partial_{\alpha, \beta} q\right)(z)-z^{\alpha-1} \bar{z}^{\beta-1}\left(S_{-1} q\right)(z),
\end{aligned}
$$


and so

$z^{\alpha-2} \bar{z}^{\beta-1}(z-\bar{z}) v(T)\left(S_{-1} f\right)(z)=z^{\alpha-2} \bar{z}^{\beta} \frac{2}{\beta i}\left(\partial_{\alpha, \beta} q\right)(z)-z^{\alpha-1} \bar{z}^{\beta-1}\left(S_{-1} q\right)(z)$.

Thus,

i.e.,

$$
(z-\bar{z}) v(T) y f(z)=\bar{z} \frac{2}{\beta i}\left(\partial_{\alpha, \beta} q\right)(z)-z\left(S_{-1} q\right)(z)
$$

$$
f(z)=-\frac{i}{2} y^{-2} v(T)^{-1}\left[\bar{z} \frac{2}{\beta i}\left(\partial_{\alpha, \beta} q\right)(z)-z\left(S_{-1} q\right)(z)\right] .
$$

The right-hand side is an axial log-polynomial sum, while the left-hand side is analytic. Thus it is in fact a log-polynomial sum. But the left-hand side approaches $a_{0}$ exponentially as $z \rightarrow \infty$ within $W_{\theta}, 0<\theta<\pi / 2$. By Lemma 5.2, then, both sides are constant.

Remark 5.2. In the sequel [Pa99], we present a Riemann-Hecke-Bochner correspondence theorem for nonanalytic automorphic integrals, together with several interesting applications.

\section{References}

[Ap90] T. M. Apostol, Modular Functions and Dirichlet Series in Number Theory, 2nd ed., Springer, New York, 1990.

[Ei57] M. Eichler, Eine Verallgemeinerung der Abelsche Integrale, Math. Z. 67 (1957), 267-298.

[ER74] J. Elstrodt und W. Roelcke, Über das wesentliche Spektrum zum Eigenwertproblem der automorphen Formen, Manuscripta Math. 11 (1974), 391-406.

[Fr85] S. Friedberg, Differential operators and theta series, Trans. Amer. Math. Soc. 287 (1985), 569-589.

[Gu63] R. C. Gunning, Lectures on Modular Forms, Ann. of Math. Stud. 48, Princeton Univ. Press, Princeton, 1963.

[Ha99] A. Hassen, Log-polynomial period functions for Hecke groups, Ramanujan J. 3 (1999), 119-151.

[He38] E. Hecke, Lectures on Dirichlet Series, Modular Functions and Quadratic Forms, Edwards Brothers, Ann Arbor, 1938.

[He59] —, Mathematische Werke, Vandenhoeck \& Ruprecht, Göttingen, 1959.

[Hu81] A. Hurwitz, Grundlagen einer independenten Theorie der elliptischen Modulfunctionen und Theorie der Multiplicatorgleichungen erster Stufe, Math. Ann. 18 (1881), 528-591.

[Iw97] H. Iwaniec, Topics in Classical Automorphic Forms, Grad. Stud. Math. 17, Amer. Math. Soc., Providence, 1997.

[Ja29] C. G. J. J acobi, Fundamenta nova theoriae functionum ellipticarum, 1829.

[Kn83] M. I. Knopp, The weight-changing operator and the Mellin transform of modular integrals, in: Lecture Notes in Math. 1013, Springer, 1983, 284-291.

[Kn86] - On the Fourier coefficients of small positive powers of $\theta(\tau)$, Invent. Math. 85 (1986), 165-183. 
[Kn89] M. I. Knopp, On the Fourier coefficients of cusp forms having small positive weight, in: Proc. Sympos. Pure Math. 49, Part 2, Amer. Math. Soc., 1989, 111127.

[Kn89a] -, Recent developments in the theory of rational period functions, in: Lecture Notes in Math. 1383, Springer, 1989, 111-122.

[Kn93] —, Modular Functions in Analytic Number Theory, 2nd ed., Chelsea, New York, 1993.

[Kn94] —, Nonanalytic Modular Integrals, unpublished lectures at Bryn Mawr College, June-July 1994.

[Kn94a] -, On Dirichlet series satisfying Riemann's functional equation, Invent. Math. 117 (1994), 361-372.

[KS96] M. I. Knopp and M. Sheingorn, On Dirichlet series and Hecke triangle groups of infinite volume, Acta Arith. 76 (1996), 227-244.

[Le64] J. Lehner, Discontinuous Groups and Automorphic Functions, Math. Surveys Monographs 8, Amer. Math. Soc., Providence, 1964.

[Le66] - A Short Course in Automorphic Functions, Holt, Rinehart, and Winston, New York, 1966.

[Ma38] H. Maass, Konstruktion ganzer Modulformen halbzahliger Dimension mit Theta Multiplikatoren in einer und zwei Variablen, Abh. Math. Sem. Hansische Univ. 12 (1938), 133-162.

[Ma64] —, Lectures on Modular Functions of One Complex Variable, Lectures on Math. 29, Tata Institute of Fundamental Research, Bombay, 1964.

[Pa98] P. C. Pasles, Convergence of Poincaré series with two complex coweights, Contemp. Math., to appear.

[Pa99] - , A Hecke correspondence theorem for nonanalytic automorphic integrals on the Hecke groups, submitted.

[Ra77] R. Rankin, Modular Functions and Forms, Cambridge Univ. Press, Cambridge, 1977.

[Ri53] B. Riemann, Collected Works, 2nd ed., Dover, 1953.

[Sc74] B. Schoeneberg, Elliptic Modular Functions: An Introduction, Springer, New York, 1974.

[Sel56] A. Selberg, Harmonic analysis and discontinuous groups in weakly symmetric Riemannian spaces with applications to Dirichlet series, J. Indian Math. Soc. 20 (1956), 47-87.

[Ser70] J.-P. Serre, A Course in Arithmetic, Springer, New York, 1970.

[Sh94] G. Shimura, Introduction to Arithmetic Theory of Automorphic Forms, Publ. Math. Soc. Japan 11, Princeton Univ. Press, Princeton, 1994.

[Si56] C. L. Siegel, Die Funktionalgleichungen einiger Dirichletscher Reihen, Math. Z. 63 (1956), 363-373.

Department of Mathematical Sciences

Villanova University

Villanova, PA 19085

U.S.A.

E-mail: pasles@e-math.ams.org

Web: http://www.angelfire.com/id/math 